\title{
A 4-week wait 'fast-track' sleep service is effective at establishing vocational drivers on continuous positive airway pressure
}

\author{
Authors: Sophie D West, ${ }^{\mathrm{A}}$ Beatrice Downie, ${ }^{\mathrm{B}}$ Gillian Olds, ${ }^{\mathrm{C}}$ Mark Tomlinson, ${ }^{\mathrm{D}}$ Claire Wotton, ${ }^{\mathrm{E}}$ Emma Firth ${ }^{\mathrm{F}}$ \\ and Alison McMillan ${ }^{\mathrm{G}}$
}

\begin{abstract}
We sought to establish whether an expedited or 'fast-track' NHS service to diagnose obstructive sleep apnoea (OSA) and establish vocational drivers on continuous positive airway pressure (CPAP) within 4 weeks of referral was possible. This model is recommended by the OSA Partnership Group. In total, 55 vocational drivers were referred to two sleep services. Assessment showed $73 \%$ had moderate or severe OSA on sleep study. Of those commenced on CPAP, review was a mean of 15 days after initiation (range 3-62 days). Median time from referral (or first clinic visit) to review on CPAP was 32 days, showing a 'fast-track' pathway is deliverable.
\end{abstract}

KEYWORDS: Continuous positive airway pressure, CPAP, fast-track service, obstructive sleep apnoea

\section{Introduction}

Sleepiness due to obstructive sleep apnoea (OSA) can impair driving, with OSA being implicated in road traffic accidents; treatment with continuous positive airway pressure (CPAP) is effective at improving sleepiness and reducing risk of crashes. ${ }^{1-4}$ However, fear of lengthy investigations and driving licence regulations may deter patients who drive for a living from seeking diagnosis and treatment for suspected OSA.

Authors: A consultant physician, Newcastle Regional Sleep Service, Newcastle upon Tyne Hospitals NHS Foundation Trust Trust, Newcastle upon Tyne, UK; ${ }^{\text {B }}$ specialty trainee, Newcastle Regional Sleep Service, Newcastle upon Tyne Hospitals NHS Foundation

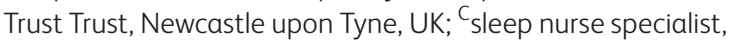
Newcastle Regional Sleep Service, Newcastle upon Tyne Hospitals NHS Foundation Trust Trust, Newcastle upon Tyne, UK; ${ }^{\text {D }}$ Sleep nurse specialist, Newcastle Regional Sleep Service, Newcastle upon Tyne Hospitals NHS Foundation Trust Trust, Newcastle upon Tyne, UK; Esleep nurse specialist, The Lister Hospital Sleep Service, East \& North Hertfordshire NHS Trust, Stevenage, UK; F sleep technician, The Lister Hospital Sleep Service, East \& North Hertfordshire NHS Trust, Stevenage, UK; ${ }^{\mathrm{G}}$ consultant physician, The Lister Hospital Sleep Service, East \& North Hertfordshire NHS Trust, Stevenage, UK
The Driver and Vehicle Licensing Agency (DVLA) leaflet Tiredness can kill - advice for drivers including drivers with obstructive sleep apnoea syndrome states that those with 'sleepiness/tiredness during the day' should contact the DVLA if they hold a current driving licence of any type. ${ }^{5}$ Recent DVLA guidance for medical professionals states that patients with excessive sleepiness, including OSA, must not drive and depending on the severity of the OSA on sleep study they either may need to notify the DVLA or must notify the DVLA; with subsequent licensing requiring medical confirmation of 'control of condition, sleepiness improved, treatment adherence'. ${ }^{6}$ This guidance may have a significant effect on the ability of vocational drivers to earn a living during the period of OSA investigation, commencement on CPAP and review.

The OSA Partnership Group is campaigning for expedited sleep services for vocational drivers, to encourage drivers to present and seek treatment. They are asking the Department of Health to issue the following guidance to clinical commissioning groups (CCGs), hospitals and GPs:

The treatment of vocational drivers with obstructive sleep apnoea syndrome (OSAS) should be expedited to allow driving again within a maximum of four weeks following first referral. ${ }^{7}$

We sought to establish whether such an expedited or 'fasttrack' service to diagnose OSA and successfully establish vocational drivers on CPAP within 4 weeks of referral was possible. Two separate NHS hospitals in England independently changed their systems and collected their results.

\section{Methods}

Newcastle Regional Sleep Service is a large teaching hospital providing CPAP for 6,000 patients; Lister Hospital Sleep Service is a district general hospital providing CPAP for 1,600 patients. At each hospital, clinic spaces were identified with the sleep nurses or sleep physiologists for patients to have an expedited initial consultation. At this appointment, assessment included evaluation of symptoms via history and the Epworth Sleepiness Score (ESS), along with same night domiciliary multichannel respiratory polygraphy (Stowood Visi Black Flash or Philips 
Alice PDx), with next day automatic download and manual review of results. If CPAP was indicated based on presence of moderate or severe OSA on the sleep study or mild OSA with symptoms compatible with a diagnosis of OSA, it was commenced on the same day or shortly after. We identified a follow-up clinic space 1-2 weeks later for review of the patient, review of symptoms, ESS and CPAP adherence via machine download. The patient could contact the sleep department helpline for telephone advice in the interim if required; at one of the centres (Lister), all patients had a telephone call at 3 days to troubleshoot any issues with CPAP.

The fast-track service was advertised to local GPs via a trust newsletter, encouraging identification of vocational drivers at the time of referral. Those who were not identified as vocational drivers at referral were fast tracked from their first routine clinic appointment at which their driving status was recognised. They had a same night domiciliary sleep study with urgent CPAP initiation and 1-week review. If the patient could not attend a 1-week review appointment, another was made as soon as they could attend thereafter.

As this was not a research study, ethical approval was not required.

\section{Results}

Between January 2014 and April 2016 in Lister Hospital and September 2014 and July 2015 in Newcastle, 30 and 29 drivers were referred, respectively, or identified as fast-track referrals; four in total failed to attend an initial appointment, so a total of 55 went through the fast-track system.

Of these, 25 held a type 2 driving licence (driving heavy goods vehicle $\mathrm{n}=12$, bus $\mathrm{n}=11$, coach $\mathrm{n}=1$, ambulance $\mathrm{n}=1$ ), 11 were taxi drivers, one a train driver and seven drove a car or van for work. This information was not recorded for 11 people. The mean age was 48.6 years (SD 9.1, range 21-65 years), mean body mass index was 35.6 (SD 6.6, range 21-51) and mean ESS was 11.6 (SD 6.1, range 0-23).

Sleep studies showed a mean $4 \%$ oxygen desaturation index (ODI) of 35 per hour (SD 30, range 0-93), meeting criteria for a diagnosis of moderate or severe OSA in 40 patients $(73 \%)$. Seven people had no significant OSA (ODI $<5 /$ hour), eight people had mild OSA (ODI 5-10/hour), ten had moderate OSA (ODI 11-20/hour) and 30 had severe OSA (ODI $>20 /$ hour).

Of the 55 drivers who attended for initial fast-track assessment, 43 were commenced on CPAP $(78 \%)$. They were reviewed a mean of 15 days after initiation (SD 10.1, range 3-62 days). Mean ESS at review on CPAP was 6.4 (SD 5.1, range $0-21)$. This fall in ESS after CPAP was significant $(\mathrm{p}<0.001)$. Mean CPAP usage was 4.9 hours/night (SD 2.3 range $0-9.2$ ). Median time from referral (or first clinic visit) to review on CPAP was 27 days (range 8-106 days). All delays in the pathway were due to patient non-attendance or patients re-scheduling appointments.

\section{Discussion}

These data demonstrate that a 4-week wait 'fast-track' service to allow driving again within a maximum of 4 weeks following first referral is deliverable within current sleep services at two different NHS hospitals with no extra resource, just reallocation of existing appointments. It can diagnose OSA in vocational drivers and successfully establish them on CPAP within a short time scale, many within 4 weeks of referral. Patients reviewed on CPAP after a mean of 15 days had a satisfactory ESS and compliance, suggesting that this is an appropriate time to evaluate their response to treatment.

It is vital that referrers are aware of the service and identify patients as vocational drivers at time of referral. We hope that other sleep centres will adopt this fast-track approach to encourage vocational drivers with suspected OSA to present for investigation and treatment, and potentially lead to safer roads.

\section{Conflicts of interest}

The authors have no conflicts of interest to declare.

\section{Author contributions}

All authors were involved in the design of this work. GO, MT, CW and $\mathrm{EF}$ were involved in data collection; $\mathrm{BD}$, SW and $\mathrm{AM}$ completed data analysis; SW wrote the final manuscript, which all authors have reviewed.

\section{References}

1 George CF. Reduction in motor vehicle collisions following treatment of sleep apnoea with nasal CPAP. Thorax 2001;56:508-12.

2 Tregear S, Reston J, Schoelles K, Phillips B. Continuous positive airway pressure reduces risk of motor vehicle crash among drivers with obstructive sleep apnea: systematic review and meta-analysis. Sleep 2010;33:1373-80.

3 Antonopoulos CN, Sergentanis TN, Daskalopoulou SS, Petridou ET. Nasal continuous positive airway pressure (nCPAP) treatment for obstructive sleep apnea, road traffic accidents and driving simulator performance: a meta-analysis. Sleep Med Rev 2011;15:301-10.

4 Stevenson MR, Elkington J, Sharwood L et al. The role of sleepiness, sleep disorders, and the work environment on heavy-vehicle crashes in 2 Australian states. Am J Epidemiol 2014;179:594-601.

5 Driver and Vehicle Licensing Agency. Tiredness can kill - advice for drivers including drivers with obstructive sleep apnoea syndrome (OSAS). IFN 159. Swansea: DVLA, 2012.

6 Driver and Vehicle Licensing Agency. Assessing fitness to drive - a guide for medical professionals. Swansea: DVLA, 2016.

7 OSA Partnership Group. Four-week wait campaign for the treatment of obstructive sleep apnoea syndrome (OSAS). OSA Partnership Group, 2015.

Address for correspondence: Dr Sophie West, Newcastle Regional Sleep Service, Freeman Hospital, Newcastle upon Tyne NE7 7DN, UK,

Email: Sophie.west@nuth.nhs.uk 